\title{
Correspondance de Fénelon, tome XVIII, Suppléments et corrections, par Jacques Le Brun, Bruno Neveu $†$ et Irénée Noye
}

Benedetta Papasogli

\section{(2) OpenEdition}

Journals

Edizione digitale

URL: http://journals.openedition.org/studifrancesi/9529

DOI: $10.4000 /$ studifrancesi.9529

ISSN: 2421-5856

\section{Editore}

Rosenberg \& Sellier

\section{Edizione cartacea}

Data di pubblicazione: 1 décembre 2007

Paginazione: 654

ISSN: 0039-2944

\section{Notizia bibliografica digitale}

Benedetta Papasogli, «Correspondance de Fénelon, tome XVIII, Suppléments et corrections, par Jacques Le Brun, Bruno Neveu t et Irénée Noye», Studi Francesi [Online], 153 (LI | III) | 2007, online dal 30 novembre 2015, consultato il 12 janvier 2021. URL: http://journals.openedition.org/studifrancesi/9529 ; DOI: https://doi.org/10.4000/studifrancesi.9529

Questo documento è stato generato automaticamente il 12 janvier 2021.

\section{cc) (†)}

Studi Francesi è distribuita con Licenza Creative Commons Attribuzione - Non commerciale - Non opere derivate 4.0 Internazionale. 


\title{
Correspondance de Fénelon, tome XVIII, Suppléments et corrections, par Jacques Le Brun, Bruno Neveu $†$ et Irénée Noye
}

\author{
Benedetta Papasogli
}

\section{NOTIZIA}

Correspondance de Fénelon, tome XVIII, Suppléments et corrections, par Jacques LE BRUN, Bruno NEVEU † et Irénée NOYE, Genève, Droz, 2007, pp. 250.

1 Dopo vari anni dalla pubblicazione degli ultimi volumi della Correspondance di Fénelon, si conclude, con questo tomo XVIII, la monumentale impresa iniziata nel 1972 e portata avanti presso due diversi editori (Klincksieck e Droz) dalla collaborazione di tre studiosi: Jean Orcibal, Jacques Le Brun e Irénée Noye. Si sarà notato che la terna di curatori di quest'ultimo volume non è più la stessa, e che il terzo nome inserito è, anche in questo caso, il nome di uno studioso scomparso: Bruno Neveu. Questo tomo di supplementi, che integra al suo interno il necessario apparato critico - mentre si ricorderà che gli altri volumi della serie erano sdoppiati regolarmente in un volume di testo e un volume di apparato -, comprende varie voci, di cui due principali: a) testi inediti, in parte ritrovati proprio da Bruno Neveu presso il fondo del Sant'Uffizio agli Archivi Vaticani, e riguardanti il processo delle Maximes des saints, mentre un altro importante lotto di lettere inedite, tra Fénelon e la Maréchale de Noailles, si trova presso le Archives nationales in Francia; b) tutto un corpus di lettere spirituali senza data e senza destinatario, già edite nell'ed. Gosselin del 1851, ma finora escluse dallo sviluppo cronologico che regge l'edizione moderna della Correspondance.

2 È quanto basta a dire l'interesse di questo tomo tardivo, che si giova di tutto un movimento di ricerca avviato intorno alle lettere di Fénelon da ormai oltre trent'anni. 
L'apertura degli Archivi del Sant'Uffizio promette nuove 'trouvailles' ed elementi che potranno offrire sulla storia del processo una luce definitiva. Colpiscono nelle lettere qui pubblicate le impressioni nettamente sfavorevoli a Fénelon da parte del nunzio Delfini; si profila sullo sfondo, con particolare evidenza, la grande ombra ostile di Luigi XIV. Ma vogliamo piuttosto attirare l'attenzione sul corpus delle lettere spirituali: sicuramente autentiche, seppure magari ritagliate e antologizzate, raggruppate a seconda dell'anonimo destinatario - così da render sensibile la sottile variazione psicologica e spirituale con cui Fénelon accompagna la storia di un'anima -, esse non sono meno belle che tante altre già note e più citate. Così ad esempio il gruppo di 27 lettere scritte a una sconosciuta, identificata con la lettera $\mathrm{Z}$, sono un piccolo denso capolavoro di direzione spirituale. L'anonimato del destinatario, l'assenza di fonti esterne per ricostruirne il profilo, impegna in un affascinante esercizio: leggere le lettere di direzione di Fénelon come lo specchio di un volto altrimenti invisibile, come una storia raccontata - non da un io omodiegetico, né alla terza persona - ma, più originalmente, alla seconda persona, all'interno di una fruttuosa relazione. E di fronte alla scarsità di notizie, l'apparato critico si fa leggero, e lascia il lettore più solo di fronte a un testo dalla risonanza più universale. Insomma questo tomo XVIII, lungi dal presentarsi come un'appendice erudita, integra l'edizione della corrispondenza con un suo apporto specifico e sostanziale. 